\title{
A Morphological Gradient Approach to Color Edge Detection
}

\author{
Adrian N. Evans and Xin U. Liu
}

\begin{abstract}
A new color edge detector based on vector differences is proposed. The basic technique gives as its output the maximum distance between the vectors within a mask. When applied to scalar-valued images, the method reduces to the classic morphological gradient. The technique is relatively computationally efficient and can also be readily applied to other vector-valued images. To improve the performance in the presence of noise, a novel pairwise outlier rejection scheme is employed. A quantitative evaluation using Pratt's figure of merit shows the new technique to outperform other recently proposed color edge detectors. In addition, application to real images demonstrates the approach to be highly effective despite its low complexity.
\end{abstract}

Index Terms-Color image processing, edge detection, morphological operations.

\section{INTRODUCTION}

$\mathbf{E}$ DGE detection is a common image processing task that often forms the initial stage of automated image interpretation, and consequently recent attention has been given to the development of color edge detection operators. The advantage of color edge detection schemes over grayscale approaches is easily demonstrated by considering the fact that those edges that exist at the boundary between regions of different colors cannot be detected in grayscale images if there is no change in intensity. Novak and Shafer [1] found that grayscale edge detection can account for $90 \%$ of the total edge points in a color image and, therefore, some form of color edge detection is required to resolve the remaining $10 \%$ of points. This approach is also compatible with that of the human visual system where color plays a significant role in the perception of boundaries.

This paper proposes a new color edge detector that has a single channel form equivalent to the morphological gradient [2] and, thus, avoids the need for any image smoothing, which is perceptually not a well-defined operation for color images. The traditional problem associated with the extension of morphological operators to multichannel images is the lack of an explicit ordering for vector quantities. For color edge detection, limited prior work exists. This includes the use of lexicographical ordering to develop a multiscale gradient operator used as part of a morphological segmentation scheme [3] and the reduced ordering (R-Ordering) employed by the vector order statistics (VOS) edge detectors of [4], [5]. Following a brief review

Manuscript received October 14, 2004; revised May 24, 2005. The associate editor coordinating the review of this manuscript and approving it for publication was Dr. Nicolas Merlet.

A. N. Evans is with the Department of Electronic and Electrical Engineering, University of Bath, Bath BA2 7AY, U.K. (e-mail: a.n.evans@bath.ac.uk).

$\mathrm{X}$. Liu was with the Department of Electronic and Electrical Engineering, University of Bath, Bath BA2 7AY, U.K. He is now with the School of Electronics and Computer Science, University of Southampton, Southampton SO17 1BJ, U.K. (e-mail: x.liu@soton.ac.uk).

Digital Object Identifier 10.1109/TIP.2005.864164 of previous work in color edge detection in Section II, the VOS edge detectors are selected for comparative purposes as they are closest in spirit to the edge detectors proposed in this paper. Results are also compared with the recently proposed compass operator [6] that provides a high performance, albeit with a high computational complexity.

The VOS edge detectors were inspired in part by the monochrome morphological edge operators of [7]. The proposed method takes as its starting point the classic morphological edge detector, given by the difference between a dilation and an erosion [2] and develops a new vector method for color edge detection termed the color morphological gradient (CMG). The CMG edge detector identifies the maximum and minimum pixels in one operation, although it does not distinguish between them. This is in contrast to the VOS edge detectors that sort the pixels in ascending order from the vector median to the vector extremum. However, the CMG edge detector suffers from the same sensitivity to noise as the classic morphological gradient and this has motivated the development of the robust CMG (RCMG) operator that employs a novel pairwise pixel rejection scheme to provide a better estimate of the true gradient in the presence of noise. The CMG and RCMG were first introduced in [8] and are described in detail in Section III.

In grayscale edge detection, the Canny edge detector [9] has become a de facto standard. This is partly because its nonmaximal suppression and thresholding with hysteresis stages produce thin, well-connected edge maps. It is, therefore, desirable to be able to apply these stages to the raw gradient of color edge detectors, an approach followed by the compass operator of [6]. To enable nonmaximal suppression to be applied to the CMG operators an estimate of the edge direction is required and this is considered in Section III-D. In addition, for nonmaximal suppression to be effective the behavior of the gradient at step edges needs to be evaluated to ensure that the maximum response coincides with the best estimate of edge location. To this end, Section III-B considers the effect of different parameter values on the RCMG behavior in the vicinity of step edges in real color images. In Section IV, a quantitative evaluation of the new operators using Pratt's figure of merit (FOM) [10] and a qualitative performance assessment on natural color images are presented. Finally, conclusions are given in Section V.

\section{COLOR EDGe Detection}

In their review paper on color image segmentation, Lucchese and Mitra divide color edge detectors into those techniques that embed the variations of all color channels in a single measure and those that compute the gradient in each channel and then combine according to certain criteria [11]. Ruzon and Tomasi [6] go further and group color edge detection methods into three categories: output fusion methods, multidimensional gradient 
methods and vector methods. Output fusion methods apply single-channel edge detection techniques to each color plane and then combine the results using, for example, a summation [12] or an OR operation [13]. A more common approach is to fuse the individual responses using some form of weighted sum [14], [15]. The appropriate color space in which to form the individual channel responses has also received attention [16]. In multidimensional gradient methods, the gradients from the individual channels are recombined before the edge decision, giving rise to a single edge estimate. Early work by Robinson applies a simple logical operation to 24 directional derivatives [17]. A more sophisticated approach after Di Zenzo [18] computes the derivatives of the gradient in the horizontal and vertical directions and the products used to form a $2 \times 2$ matrix in each component. The matrices are then summed over all channels and the edge magnitude and direction given by the principal eigenvalue and the related eigenvector, respectively. Variations of this approach have been used by Cumani [19], Chapron [20], and Alshatti and Lambert [21]. A comparison of these and other, simpler techniques is presented in [22]. An alternative approach to the problem of fusing the gradients is the use of Dempster-Shafer theory [23].

The main problem with both output fusion and multidimensional gradient methods is how to combine the channels to give a final result. Therefore, it is the final class of techniques, vector methods, that offers the greatest potential. Previous research into vector methods has used differential geometry to determine the rate of change and corresponding direction at each point [24], [25]. Other research has considered the use of probability distributions. For example, Fotinos et al. propose the use of relative entropy as a dissimilarity measure between a local probability distribution and that of a homogenous region [26] while Ruzon and Tomasi prefer the use of color signatures generated using vector quantization [6]. Alternatively, a vector difference algorithm can be used. Wen et al. use a vector form of the Sobel operator to determine the edge strength in four directions and then apply a threshold to the direction with the maximum response [27]. In a similar manner, the output of the vector gradient edge detector is the maximum vector gradient across the central pixel [28] and the LUV gradient of [29] calculates the greatest distance between the central pixel and its nearest neighbors in LUV space. In addition to evaluating the edge magnitude, these approaches are, therefore, also able to provide an estimate of edge direction. The VOS edge detectors [4], [5] employ R-Ordering to sort a set of vectors according to their aggregate distances to all other vectors in the set. The simplest VOS operator is the vector range (VR) edge detector that measures the distance between the lowest and highest ranked vectors, i.e. the vector median and the vector extremum, respectively. Several other VOS operators were proposed to increase the robustness to noise, of which the minimum vector dispersion (MVD) was shown to be the most effective. However, in contrast with the previous vector edge methods, the MVD is unable to provide an estimate of edge direction.

\section{COLOR MORPhOlOGiCAl GRADIENT OPERATORS}

The classic morphological gradient operator for grayscale images is the difference between a dilation and an erosion [2] and, for a structuring element $g$, is given by

$$
\nabla(f)=\delta_{g}(f)-\epsilon_{g}(f) .
$$

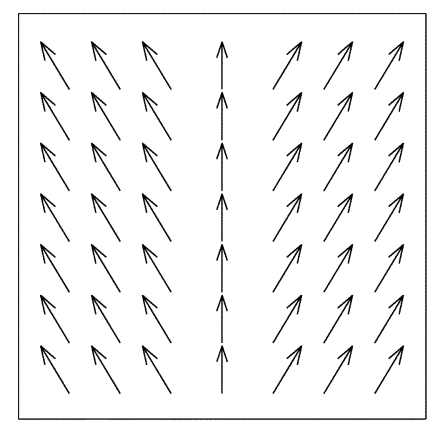

(a)

\begin{tabular}{|lllllll|}
\hline 0 & 0 & $\mathrm{R} / 2$ & $\mathrm{R}$ & $\mathrm{R} / 2$ & 0 & 0 \\
0 & 0 & $\mathrm{R} / 2$ & $\mathrm{R}$ & $\mathrm{R} / 2$ & 0 & 0 \\
0 & 0 & $\mathrm{R} / 2$ & $\mathrm{R}$ & $\mathrm{R} / 2$ & 0 & 0 \\
0 & 0 & $\mathrm{R} / 2$ & $\mathrm{R}$ & $\mathrm{R} / 2$ & 0 & 0 \\
0 & 0 & $\mathrm{R} / 2$ & $\mathrm{R}$ & $\mathrm{R} / 2$ & 0 & 0 \\
0 & 0 & $\mathrm{R} / 2$ & $\mathrm{R}$ & $\mathrm{R} / 2$ & 0 & 0 \\
0 & 0 & $\mathrm{R} / 2$ & $\mathrm{R}$ & $\mathrm{R} / 2$ & 0 & 0 \\
\hline
\end{tabular}

(b)
Fig. 1. (a) Ideal color ramp edge consisting of vectors $V_{1}=(-3,-5), V_{2}=$ $(3,-5)$, and $V_{3}=(0,-5)$. (b) CMG response where $R=\left|V_{1}-V_{2}\right|=6$ and $R / 2=\left|V_{1}-V_{3}\right|=3$.

The starting point in the development of the CMG is to express (1) in the alternative form

$$
\begin{aligned}
\nabla(f) & =\max _{x \in g}\{f(x)\}-\min _{y \in g}\{f(y)\} \\
& =\max (|f(x)-f(y)|) \forall x, y \in g .
\end{aligned}
$$

Expressed in this form, the morphological gradient is given as the greatest absolute intensity difference between any two pixels within the structuring element and can easily be extended to color images. Let $\mathcal{X}=\left[\mathbf{X}^{1}, \mathbf{X}^{2}, \cdots, \mathbf{X}^{n}\right]$ be the set of $n$ vectors contained within a structuring element $g$. The CMG $\nabla(\vec{f})$ can then be defined as

$$
\nabla(\vec{f})=\max _{i, j \in \mathcal{X}}\left\{\left\|\mathbf{X}^{i}-\mathbf{X}^{j}\right\|_{p}\right\}
$$

whose response is the maximum of the distances between all pairs of vectors in the set using, for example, the $L_{2}$ norm of $p=2$ to give the Euclidean distance. The vector pair that gives rise to maximum distance can be thought of as containing the maximum and minimum vectors, although no one vector can unambiguously be identified as either.

The operation of the CMG can be illustrated by considering its response to an ideal ramp edge using a $1 \times 3$ mask (see Fig. 1). Here, the response is the difference between the left and right pixels, an output that is identical to the centered difference calculated by a $\left[\begin{array}{lll}-1 & 0 & 1\end{array}\right]$ mask. Although the width of the edge response is 3 pixels wide, the true edge position is marked by the maximum response $R$ and can be found by appropriate thresholding. In contrast, the VR edge detector measures the difference between the median (center) and extremum pixels which is a noncentered difference and, for Fig. 1(a), produces a 3-pixel-wide response of $R / 2$.

The computational complexity of the $\mathrm{CMG}$, like that of the VOS operators, is $O\left(n^{2}\right)$ as it is dominated by the $(1 / 2) n(n-1)$ vector distances that have to be calculated. An efficient sliding window algorithm can be employed to reduce the complexity to $O\left(n^{3 / 2}\right)$ [5]. However, the only additional operation required for the $\mathrm{CMG}$ is determining the maximum distance so it is less complex than the MVD which also needs to update its aggregate distances, in addition to several other operations.

\section{A. Robust Color Morphological Gradient}

The CMG suffers from the disadvantage of being very sensitive to outliers due to the fact that its magnitude is derived 
from the two vectors in the window that are furthest apart. In addition, in the presence of noise and for nonideal edges, simply selecting the two extrema vectors may produce an edge response that is not necessarily representative of the true gradient. Linear edge detectors such as the Prewitt and Sobel address this problem by smoothing, to give an averaged centered difference. As smoothing is not a well-defined operation on color images an alternative approach based on pairwise pixel rejection is proposed that aims to provide a better estimate of the color gradient at each pixel position by making a more propitious choice of the two vectors used for the gradient estimate.

The pairwise pixel rejection scheme works by removing the two pixels that are furthest apart and then finding the CMG of the remaining pixels. This process can be repeated a number of times until a good estimate of the edge strength is obtained-the problem of exactly how many vector pairs to remove is considered below. The resulting process is less affected by image noise and so is known as the RCMG, $\nabla(\vec{f})_{\text {Robust }}$, and is defined by

$$
\nabla(\vec{f})_{\text {Robust }}=\max _{i, j \in\left\{\mathcal{X}-\mathcal{R}_{s}\right\}}\left\{\left\|\mathbf{X}^{i}-\mathbf{X}^{j}\right\|_{p}\right\}
$$

where $\mathcal{R}_{s}$ is the set of $s$ pairs of vectors removed.

A simple illustration of the underlying theory behind its operation is given by considering the bimodal distribution of intensities for a single channel step edge corrupted by Gaussian noise. The morphological gradient is the difference between the maximum and minimum pixels and is not representative of the difference between the two modes of the distribution. As successive pairs of pixels are removed, one from either end of the distribution, the output will move toward the difference between the two modes; the RCMG applies exactly the same operation to the vector populations associated with color images. The process should, in theory, be relatively robust to the exact number of pairs removed as the majority of the pixels have intensities on or close to the two modes. It should be noted that, in common with many other vector difference-based color edge detectors, this analysis is based on the underlying assumption that the mask contains only two populations and the estimate may be unreliable if more than two populations are present.

Applying the RCMG with a $3 \times 3$ mask at the center of the ideal color ramp edge of Fig. 1(a) produces a response of $R$, where $R=|(-3,-5)-(3,-5)|=6$, for $0 \leq s \leq 2$. However, when the ideal edge is perturbed by short-tailed (Gaussian) noise the RCMG output varies with $s$. Fig. 2 presents an $x y$ plot of pixels from the ideal edge corrupted by Gaussian noise showing the RCMG outputs for $s=0,1$ and 2 of $R_{0}=6.6765$, $R_{1}=6.0245$, and $R_{2}=5.6676$, respectively. Here, it can be seen that the distance $R_{1}$, given by setting $s=1$ in (4), provides the measure that is closest to the true gradient across the edge. The aim of the this pairwise pixel rejection is, therefore, to find a "median" or mid-ranked centered difference across the edge rather than the Sobel-like mean centered difference, an approach that does not require any vector smoothing.

The pairwise pixel rejection scheme, therefore, makes the RCMG more robust to Gaussian noise than the CMG and its performance in the presence of impulsive noise is now considered. A standard approach to impulsive noise is to employ some form of outlier rejection. For example, the MVD color edge detector uses R-ordering to effectively reject all but one of the $k$ highest ranked pixels. The pairwise pixel rejection scheme described above provides a simple and effective alternative approach to

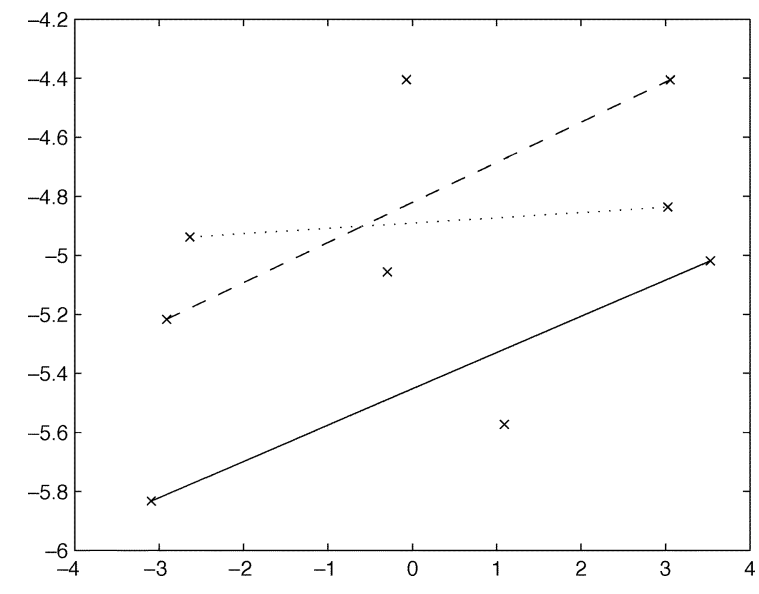

Fig. 2. RCMG example for ideal color ramp edge of Fig. 1(a) corrupted by Gaussian noise using $3 \times 3$ mask. The RCMG outputs for $s=0,1$, and 2 (solid, dashed, and dotted lines, respectively) are 6.6765, 6.0245, and 5.6676.

outlier rejection. The justification for this approach is as follows. If either or both of the removed vectors are outliers their removal is desirable while if a nonoutlying vector is removed it will have little effect on the output of the RCMG as other, similar vectors will exist.

In summary, the pairwise pixel rejection scheme employed by the RCMG is attractive as it is robust to Gaussian and impulsive noise, provides a good estimate of the true gradient and is also relatively computationally inexpensive. It has only one parameter $s$ that needs to be set and its determination is discussed below. The computational complexity of the RCMG is only slightly greater than for the CMG as its only additional computations are associated with rejecting some of the vector differences already calculated.

\section{B. Determination of RCMG Parameter Value Range}

In this section, the problem of determining an appropriate range for $s$, the number of pairs of vectors to remove in (4), is considered. For the ideal color ramp edge of Fig. 1(a), with a $3 \times 3$ mask, only one pair of vectors needs to be removed for the RCMG output to be the median centered difference. Likewise, for a $5 \times 5$ mask, $s=4$. This sets a theoretical lower limit on the number of pairs that should be removed. In real color images, edges are rarely ideal and are often corrupted by impulsive noise, and, in practice, a better performance may be achieved by removing outlying pairs of vectors before finding the median of the remaining centered differences across the edge. For a $3 \times 3$ mask, only two pairs of vectors can be removed if at least 1 pixel from the minority population is to be retained. Similarly, for a $5 \times 5$ mask with a minority population of $10 s$ should be $\leq 9$. This sets a theoretical upper limit on the number of pairs that can be removed while still producing a satisfactory performance. Therefore, the theoretical bounds for a $5 \times 5$ mask are $4 \leq s \leq 9$.

To verify if these are appropriate limits for $s$ in application to real color images, the RCMG was applied to the $512 \times 512$ color test image Lenna. Fig. 3(a) shows the image with the two edges marked in black and white. These edge positions were chosen as they produced representative results. Fig. 3(b) and (c) shows the RCMG responses across the black and whites edges, respectively, for different values of $s$ obtained using a $5 \times 5$ mask. The RCMG results for $s=0$ are included as they are equivalent to 


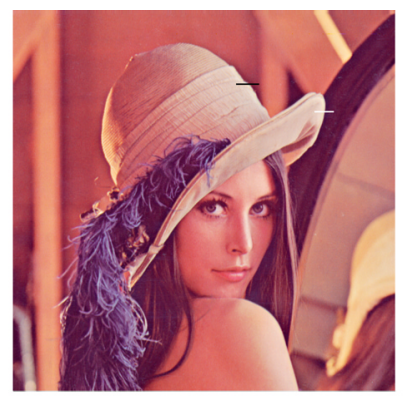

(a)

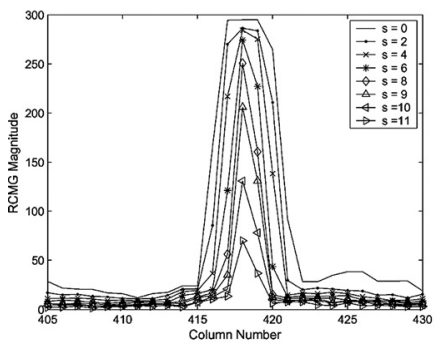

(b)

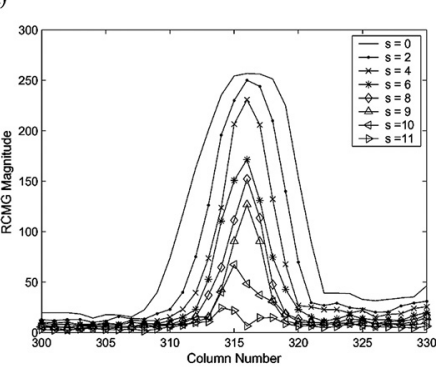

(c)
Fig. 3. Behavior of RCMG edge detector at step edges in Lenna for range of values for $s$. (a) Original image with marked edges. (b) RCMG response at black edge. (c) RCMG response at white edge. (Color version available online at http://ieeexplore.ieee.org.)

the CMG. For both edges, as $s$ increases the profile of the edge becomes better defined, enabling thinner edges to be produced by thresholding. A good edge profile is produced when $s \geq 4$ in both cases. On some edges, for example, Fig. 3(b), the RCMG operator keeps working until $s=11$ while at others, such as Fig. 3(c), the position of maximum response and edge profile degenerates for $s>9$. At all edge positions, the response of the RCMG rapidly decreases for $s>9$. Thus, for a $5 \times 5$ mask, $s$ should be less than 10 and similar experiments using a $3 \times 3$ mask have found $s$ should be less than or equal to 2 . These results are in agreement with the theoretical analysis presented above. In practice, the specific value for $s$ within this range will depend on the type and level of noise present in the image and this is the subject of an objective evaluation in Section IV-A.

\section{Color Models and Distance Metrics}

Most color images are captured using a sensor array that responds to the primary colors. Consequently, the trichromatic RGB color space is widely used in color image processing and has the advantage of requiring no color space conversion. To assess the difference between two vectors a vector norm, usually the Euclidean distance given by the $L_{2}$ norm, is probably the most popular metric. Although often used, the performance of the combination of the RGB color space and the Euclidean distance is known to be less than ideal. Other approaches that have been considered include making the distance measures in alternative color spaces. For example, the LUV color space was specifically designed so that the perceptual difference between any two colors is accurately measured by the Euclidean distance, and this was the combination used by the LUV gradient of [29]. Other authors have compared the performance of color edge detectors in a number of color spaces [16]. Alternatively, improved results can be achieved by applying metrics that correspond more closely with human perception directly in the RGB color space. This avoids the need for transforming the color space which in certain cases, for example RGB to HSI or LUV, can be computationally expensive. The idea of using the angle between vectors was introduced by the vector directional filters of [30] and provides the basis for hue-based metrics that have the ability to detect edges when the intensity is either low or unchanging. To capture the advantages of both angle and distance, combined metrics can be used [28]. One such combined metric originally proposed by Androutsos et al. [31] for use in color image retrieval and given by

$$
d_{i, j}=1-\left[1-\frac{2}{\pi} \cos ^{-1}\left(\frac{\mathbf{X}^{i} \cdot \mathbf{X}^{j}}{\left|\mathbf{X}^{i}\right|\left|\mathbf{X}^{j}\right|}\right)\right]\left[1-\frac{\left|\mathbf{X}^{i}-\mathbf{X}^{j}\right|}{\sqrt{3 \cdot 255^{2}}}\right]
$$

where the terms in the square brackets measure angle and magnitude, respectively, can easily be used in place of a norm to calculate the distances required for the $\mathrm{CMG}$ and RCMG edges detectors. The resulting combined metric form of the RCMG is given by

$$
\nabla(\vec{f})_{\text {Robust }_{\mathrm{COM}}}=\max _{i, j \in\left\{\mathcal{X}-\mathcal{R}_{s}\right\}}\left\{d_{i, j}\right\}
$$

and is applied directly in the RGB color space.

\section{Edge Direction and Thinning}

Good edge detectors produce thin, continuous lines, two aspects that are addressed by the nonmaximal suppression and threshold with hysteresis stage of the Canny edge detector [9]. The direction of maximum gradient for the CMG operators can be defined by a line joining the pixel positions of the two vectors that constitute the pair that are furthest apart; this approach follows in spirit that of the LUV gradient [29]. Although the line does not necessarily pass though the mask center, for a $5 \times 5$ mask, the worst case is only 2 pixels from the center. The line represents the maximum gradient within the mask and is, therefore, a valid measure to attribute to the mask center. As each of the pixels comes from the population on opposite sides of the edge an approximate edge direction can be given by the normal to this line.

Fig. 4 shows an example of direction variation with $s$ for the edges marked in black and white in Fig. 3(a) whose true edge directions are approximately $-45^{\circ}$ and $90^{\circ}$, respectively. Providing the two pixels used to compute the direction lie on either side of the edge the absolute direction error should be $\leq 45^{\circ}$. This is the case for virtually all direction estimates for $s \leq 9$. There are also many values of $s$ for which the direction estimate is much closer to the true value, a result that is repeated at other pixel positions. Therefore, although the RCMG cannot give a highly accurate estimate of the edge direction, it is capable of providing an approximate direction that is suitable for the nonmaximal suppression stage of the Canny edge detector which, for some implementations, simply requires the directions to be quantized into one of four quadrants. In this scenario, the real test of the quality of the edge direction estimates is the performance of the RCMG with the nonmaximal suppression scheme, which is considered in Section IV-B.

For nonmaximal suppression to work effectively, it is also important that the maximum response occurs at the center of the edge. Examination of Fig. 3(b) and (c), the RCMG responses to the step edges marked in Fig. 3(a), shows that for $s \leq 9$ the maximum RCMG response for both edges is at the center of the 


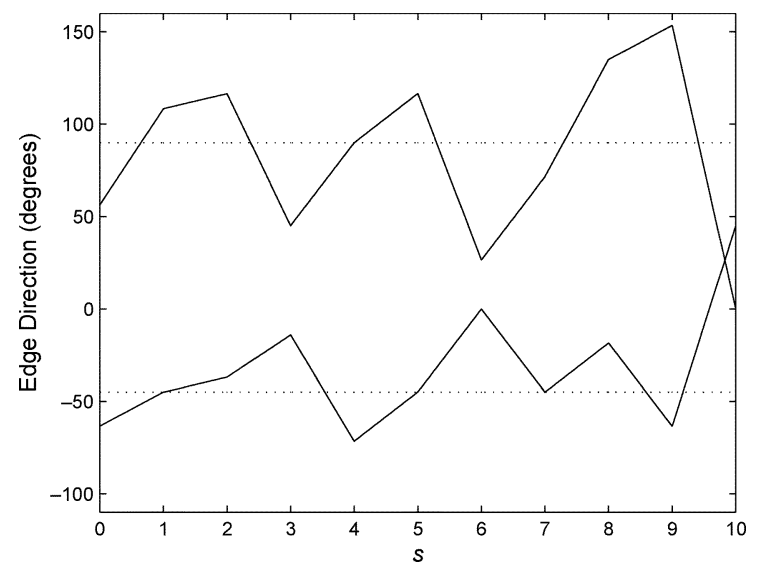

Fig. 4. Direction of edges marked in black and white in Fig. 3(a) with varying $s$. True edge directions of $-45^{\circ}$ and $90^{\circ}$, respectively, are shown with dotted lines.

edge. These result were typical of those at other edge positions in the image.

\section{EXPERIMENTAL RESULTS}

The new techniques are evaluated both quantitatively and qualitatively. The quantitative evaluation is performed using a synthetic image in conjunction with a measure of edge deviation and is used to evaluate the performance of the RCMG edge detector for different values of the parameter $s$. For comparison, the MVD edge detector [5] is used, as it conceptually close to the RCMG and has been described as the most compelling vector method of color edge detection [6]. The recently proposed compass operator of Ruzon and Tomasi [6] is also chosen as it represents the state of the art in color edge detection. However, its underlying theory and greatly increased complexity mean that as a technique it is not directly comparable with the RCMG in the same manner as, for example, the VOS operators of [4] and [5].

A subjective evaluation of the performance of the color edge detectors is undertaken using standard color test images, again using the MVD and compass operators for comparison. Both the subjective and objective results initially use the RCMG with the Euclidean distance metric applied directly to the RGB color model and a final subjective evaluation is performed to determine the benefits of the combined distance metric detailed in Section III-C. A $5 \times 5$ mask was used for all experiments as it provides a reasonable compromise between performance and complexity, although we have found that the RCMG achieves reasonable results with a $3 \times 3$ mask in many cases but with less robustness to noise.

\section{A. Quantitative Evaluation}

A quantitative evaluation of color edge detectors is problematic and when it is employed differing criteria can produce different results. The approach adopted here is to use the ground truth provided by a simulated image in conjunction with the most widely used measure of edge deviation, Pratt's FOM [10]. The FOM is defined by

$$
\mathrm{FOM}=\frac{1}{\max \left\{I_{D}, I_{I}\right\}} \sum_{k=1}^{I_{D}} \frac{1}{1+\alpha\left(d_{k}\right)^{2}}
$$

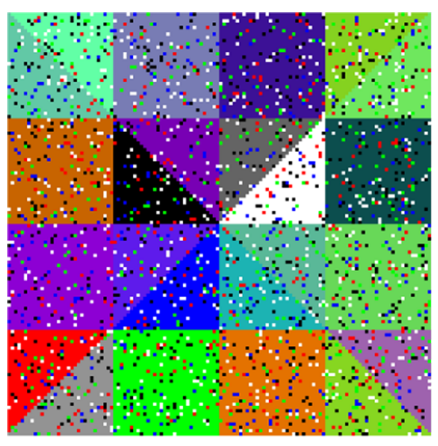

(a)

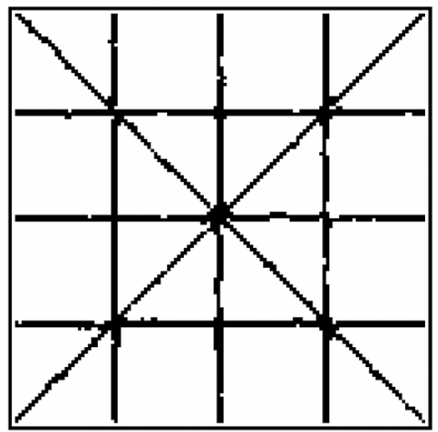

(c)

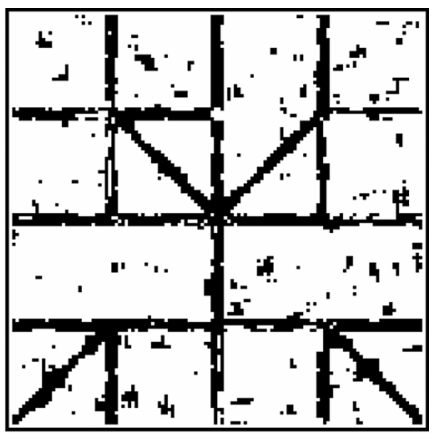

(b)

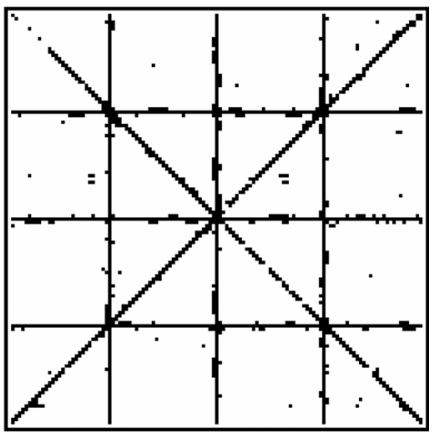

(d)
Fig. 5. Simulated color test image and color edge detector results for $15 \%$ correlated ( $\rho=0.5$ ) impulsive noise, corresponding FOM values shown bracketed. (a) Original image. (b) MVD result (0.7789). (c) RCMG result (0.9810). (d) Compass result (0.9707). (Color version available online at http://ieeexplore.ieee.org.)

where $I_{I}$ and $I_{D}$ are the number of ideal and detected edge points, respectively, and $d_{k}$ is the separation distance of the $k$ th detected edge point normal to a line of ideal edge points. The scaling constant $\alpha(>0)$ provides a relative penalty between smeared and isolated, offset edges and was set to $\alpha=0.2$. A $\mathrm{FOM}=1$ corresponds to a perfect match between the ideal and detected edge points and as the deviation of the detected points increases, the FOM approaches zero. A $128 \times 128$ simulated test image was designed to meet the requirements detailed in [5] and is shown in Fig. 5(a). The FOM for each color edge detector requires an ideal edge map and these are given by simulated edge maps corresponding to the ideal theoretical responses.

The FOM results for various levels of Gaussian and impulsive noise, both independent and correlated with a correlation factor $\rho=0.5$, produced by the RCMG edge detector for different values of the parameter $s$ are given in Fig. 6. The figures were obtained by adjusting the edge threshold for each noise level until the maximum FOM value was found. For all types of noise a value of $s=0$, corresponding to CMG edge detector, produces the worst FOM results. This is not surprising as the CMG can respond to a single noise pixel. Examination of the FOM plots for independent and correlated Gaussian noise shows that for $s \leq 4$ the results are significantly lower than for those when $s>4$ for signal-to-noise ratios (SNRs) of $13 \mathrm{~dB}$ and above. The performances for $6 \leq s \leq 8$ are broadly similar with $s=9$ performing less well. The FOM results for impulsive noise show the same pattern for both independent and correlated noise with the performance increasing with $s$ up to $s=8$. Eliminating nine pairs of vectors produces the best FOM result for very low 

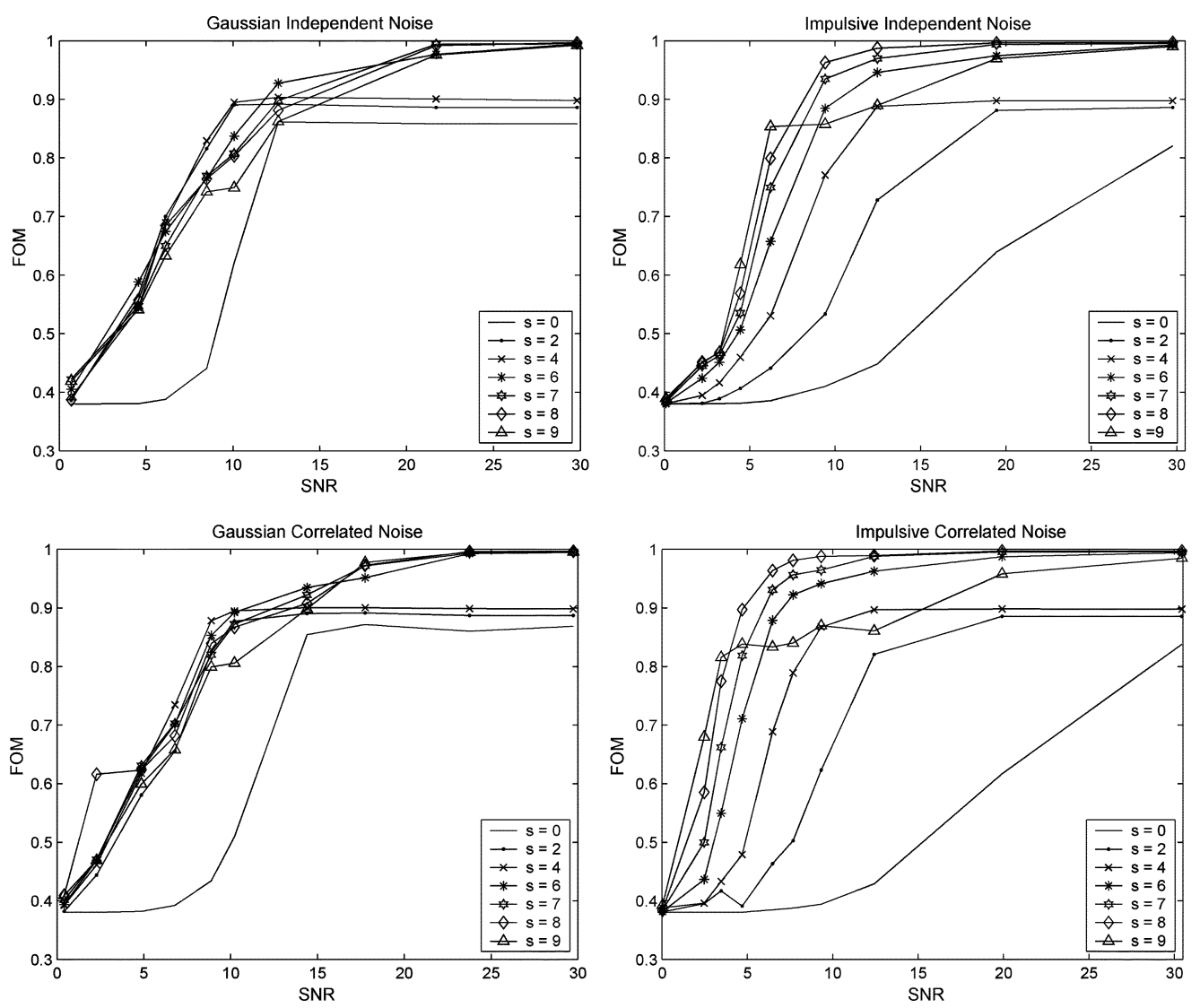

Fig. 6. FOM results for RCMG edge detector for range of values for $s$.

SNRs but is less competitive for higher SNRs. Overall, values in the range $6 \leq s \leq 8$ all give satisfactory performance and for subsequent experiments $s=8$ is chosen, as it produces the best all-round performance.

Fig. 7 compares the RCMG results for $s=8$ with those of the MVD and compass edge detectors. The range of parameter values given for the MVD in [4] are $7 \leq k \leq 10$ and $10 \leq l \leq$ 15 while in [32] $k=6$ and $l=10$. Here, values of $k=7$ and $l=10$ are chosen as they are close as possible to those of [32] while within the range of [4]. The compass operator is circular with radius $3 \sigma$ and a value of $\sigma=0.94$ was chosen so that its area was 25 , equal to that of the $5 \times 5$ masks used by the other operators.

For Gaussian independent noise, the FOM performance for all detectors is broadly similar, although it can be seen that the RCMG produces a superior result for the majority of cases. When the Gaussian noise is correlated the RCMG has the highest FOM with the exception of SNRs around $14.5 \mathrm{~dB}$, with the greatest advantage for SNRs below $14 \mathrm{~dB}$. For impulsive noise, the FOM performance of the RCMG is markedly better than the MVD for both independent and correlated noise; for both cases, the FOM of the RCMG is above that of the MVD for the entire $0-30 \mathrm{~dB}$ range. The compass operator out-performs the RCMG for low SNRs, in particular, for independent impulsive noise. However, above $9.4 \mathrm{~dB}$ for impulsive independent noise and $6.4 \mathrm{~dB}$ for impulsive correlated noise, the FOM performance of the RCMG edge detector is at least equal to, and, in some cases, slightly above, that of the compass operator despite its greatly reduced complexity.

The performance advantage of the RCMG is further illustrated by the edge detection results for $15 \%$ of correlated $(\rho=$
0.5) impulsive noise corresponding to a SNR of $7.67 \mathrm{~dB}$, see Fig. 5. At this noise level, the FOM for the MVD is only 0.7789 , reflecting the many noise points and missing true edge points that can be seen in Fig. 5(b). This result also shows that the MVD produces a double response to a single edge with edge points marked either side of a missing true edge point, something first reported in [8]. Fig. 5(c) clearly demonstrates the robustness of the RCMG detector which shows little degradation due to noise and this is confirmed by a FOM of 0.9810 . The compass result of Fig. 5(d) has thinner edges than that of the RCMG by virtue of its nonmaximal suppression but also exhibits more noise responses, resulting in a slightly lower FOM of 0.9707 .

\section{B. Natural Color Images}

Despite the usefulness of objective measures, the subjective performance of color edge detectors remains a fundamental component of results interpretation. In this study, a number of different color test images have been evaluated and the results presented here for the Peppers test image are representative of those obtained. Fig. 8(a) presents the original color image with four regions of interest marked by three black ellipses and one white rectangle. Fig. 8(b)-(d) presents the unthresholded gradient magnitude responses of the MVD, RCMG, and compass edge detectors, respectively. The parameters used were $k=7$ and $l=10$ for the MVD, $s=8$ for the RCMG, and $\sigma=0.94$ for the compass operator, as before. The RCMG produces thinner, more continuous edges than the MVD. To illustrate this point, Fig. 9 presents closeups of the central green pepper and shows the superior performance of the RCMG edge detector, and the double-peaked response of the MVD detector. The result produced by the compass operator in Fig. 8(d) shows 

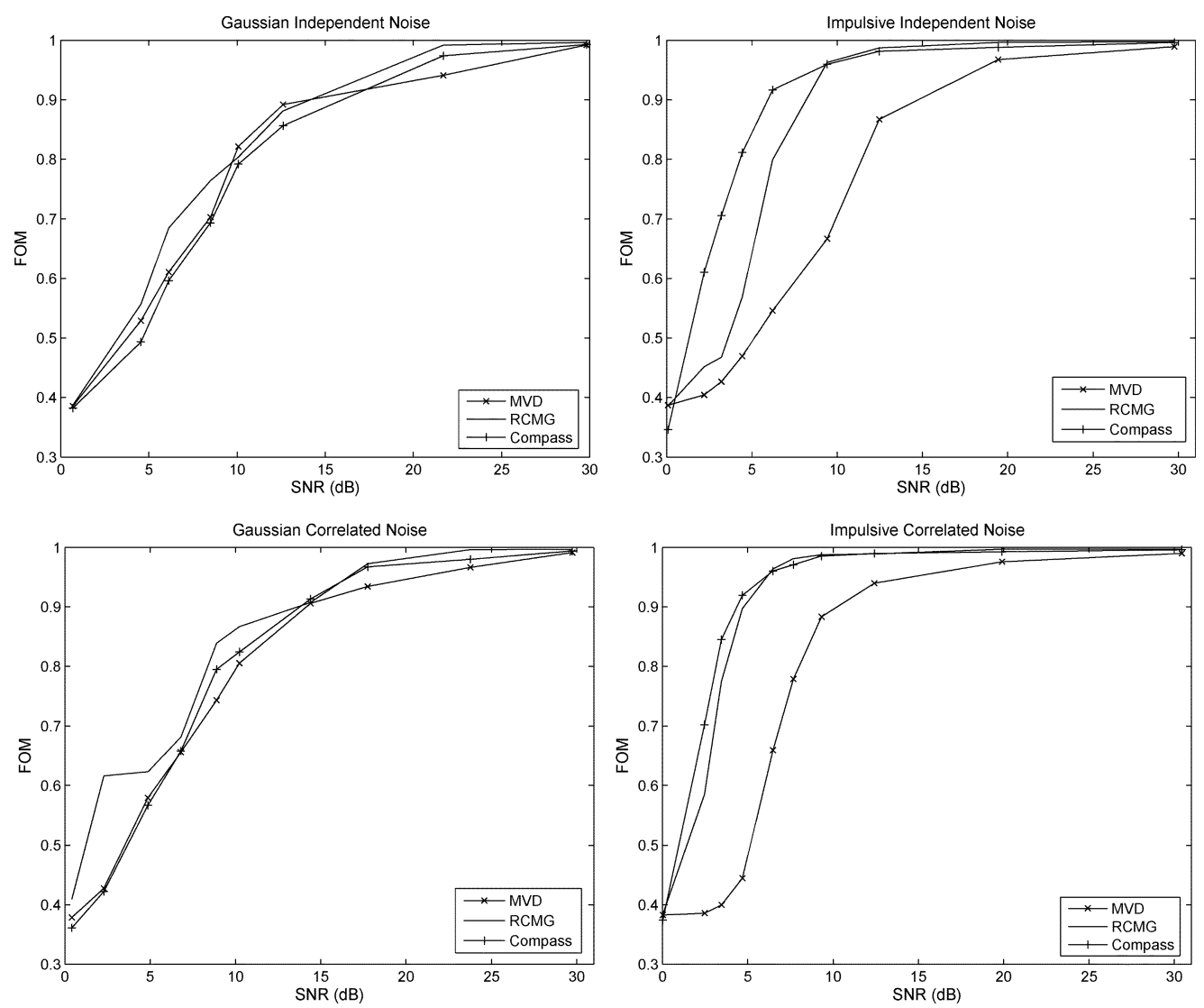

Fig. 7. FOM results for RCMG, MVD, and Compass color edge detectors.

a strong response to color edges in areas of low intensity, for example in the region marked by the white rectangle in Fig. 8(a). However, as the compass operator responds strongly to variations in color when the intensity is low, in this region, it is difficult to distinguish between the responses associated with the edge of the pepper and those due to the random color fluctuations. In comparison, it produces a relatively weak response to the edges in regions marked by black ellipses in Fig. 8(a) where there is little change in color.

Section IV-A proposed a RCMG parmeter range of $6 \leq s \leq$ 8. To help determine the most appropriate value from this range for natural images a simple threshold was applied to the RCMG responses to the Peppers image using $s=6$ and $s=8$ and the threshold value interactively adjusted to achieve the best subjective results (see Fig. 10). Of the two results, Fig. 10(b) is preferable as it has thinner edges and better edge preservation, for example in the region marked by the rightmost ellipse in Fig. 8(a). These noise free results can be used to provide the ideal edge images required by the FOM, and Fig. 11 presents the corresponding FOM plots for $6 \leq s \leq 8$. Unlike the simulated image, these FOM values can not provide an absolute quality measure as they use ideal edge maps that are both different and subjectively chosen. However, they can provide information on relative robustness. For independent and correlated Gaussian noise, the RCMG performance for all values of $s$ is very similar with the best value of $s$ changing with SNR. For impulsive noise, the FOM for $s=8$ shows the least reduction for both independent and correlated impulsive noise. From these results, it is recommended that $s=8$ should be the RCMG parameter setting for natural images.
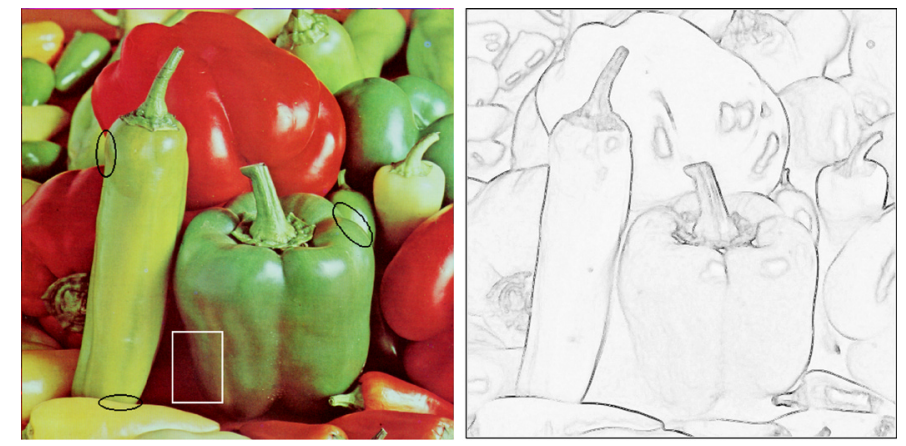

(a)

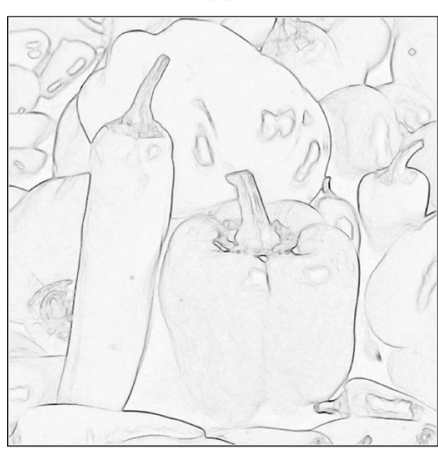

(c) (b)

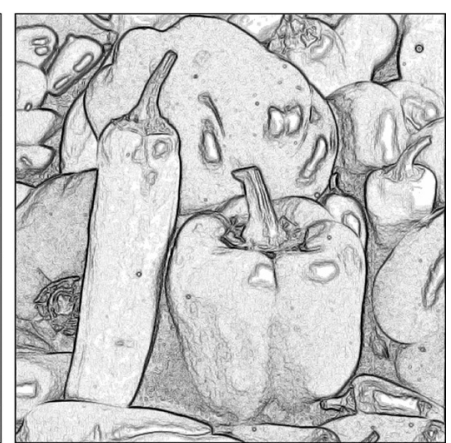

(d)
Fig. 8. Color edge results for the Peppers image (regions of interest marked by black ellipses and a white rectangle). (a) Original image. (b) MVD result. (c) RCMG result. (d) Compass result. (Color version available online at http://ieeexplore.ieee.org.) 

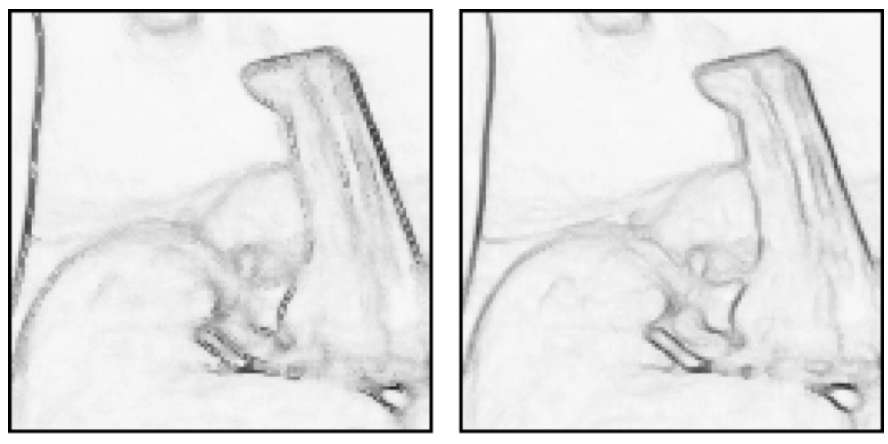

(a)

(b)

Fig. 9. Color edge results for the Peppers image. (a) Closeup of Fig. 8(b). (b) Closeup of Fig. 8(c).

Fig. 11 also shows the FOM results for MVD and the compass operators, again subjectively thresholding the noise-free gradients of each operator to produce the ideal edge map. The results follow the same general trend as those of the simulated image except the advantage of the compass operator is restricted to lower SNRs. However, for the reason stated above, the FOM analysis does not provide an absolute quality measure. Instead, Figs. 12 and 13 show the responses of the color edge detectors to the Peppers image corrupted with correlated $(\rho=0.5)$ impulsive and Gaussian noise, respectively. Comparison of Fig. 12 with the noise-free responses of Fig. 8 shows the performance of the RCMG to be only slightly affected by this level of impulsive noise. In contrast, the MVD and compass results are more degraded and show many spurious noise responses. The correlated Gaussian noise results in Fig. 13 are more degraded for all color edge detectors which is to be expected as Fig. 11 shows that Gaussian noise produces a greater FOM reduction than impulsive noise at the same SNR. The RCMG response to Gaussian noise is, on average, stronger than that of the MVD. Despite this, we have found that it produces a better edge map after thresholding as its response to true edges is stronger and more continuous. The compass result also exhibits strong, continuous edges but responds very strongly to noise in regions characterized by low intensity or relatively constant color.

As a final test, the RCMG edge results are thresholded and compared to those of the compass operator (see Fig. 14). Applying a simple threshold to the RCMG response (see Fig. 10) does not produce a suitable edge map for comparison with the compass operator as the latter produces single pixel thickness edges. To make the comparison more valid, nonmaximal suppression and thresholding with hysteresis (after Canny [9]) are applied to the RCMG gradient, using the definition of edge direction described in Section III-D. Fig. 14(a) and (b) shows the edge results of the RCMG and compass operators, respectively, after nonmaximal suppression and hysteresis thresholding. Comparing these results, the RCMG is able to detect the intensity-dominated edges in the regions marked by ellipses in Fig. 8(a) but does not detect as many edge points in the darker regions. Overall, the RCMG provides a competitive result with reduced complexity. In addition, the continuity of edges in Fig. 14(a) shows the effectiveness of the edge direction estimation process described in Section III-D.

To show the effect of using a different distance metric, the raw gradient and edge points found using the combined distance form of the RCMG given by (6) are presented in Fig. 14(c) and

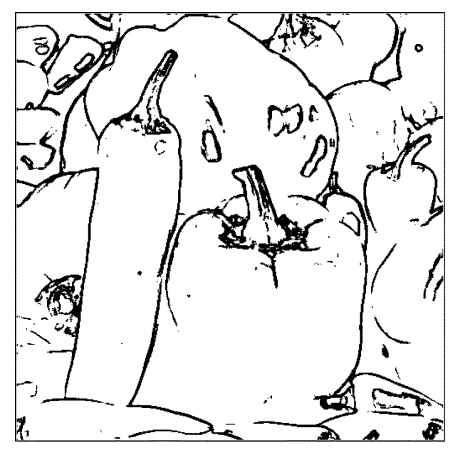

(a)

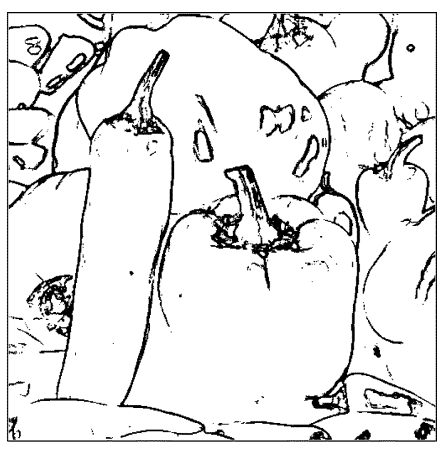

(b)
Fig. 10. Thresholded RCMG results for the Peppers image. (a) RCMG result for $s=6$, threshold $=50$. (b) RCMG result for $s=8$, threshold $=35$.

(d), respectively. These results show that the combined metric has the desired effect, strengthening the edge response in the low intensity regions of the image with relatively little negative impact elsewhere. In Fig. 14(d), the majority of edges in the regions marked by ellipses are retained and the bottom of the central green pepper in the region marked by the rectangle is now detected.

\section{CONCLUSION}

A new class of color edge detectors has been proposed that uses a morphological approach to provide an estimate of the color gradient. The method is equal to that of the classic morphological gradient when the operators are reduced to a single channel form. The operators are also computationally efficient with similar complexity to the VOS color edge detectors. The CMG is sensitive to image noise and this problem is addressed by the development of the RCMG. The RCMG successively eliminates pairs of vectors that give rise to the maximum distance, providing a good estimate of the true color gradient by using a "median" centered difference approach that requires no image smoothing.

A quantitative and qualitative study of the performance of the new operators using simulated and natural color images shows them to produce a well-behaved response that marks the center of the edge with a single maximum. Quantitative results obtained using a simulated test image corrupted by noise show the RCMG to be very robust down to low SNRs, in particular for impulsive noise. Application to real color images further demonstrates this robustness. The performance of the RCMG at the scales used is superior to that of the MVD edge detector and comparable to the compass operator, despite its lower complexity.

To enable the generation of edge maps consisting of singlepixel edges, a workable definition of edge direction for the CMG operators has been given that facilitates the application of a nonmaximal suppression stage prior to thresholding. Finally, the use of a combined distance metric has been shown to enable the RCMG to produce an output comparable with that of the compass edge detector and this is a potent area for further investigation.

Color edge detection is an important first stage of color image analysis. The $\mathrm{CMG}$ operators presented here require no conversion from the RGB color space and have been demonstrated to have good localization and noise immunity properties. As such, 

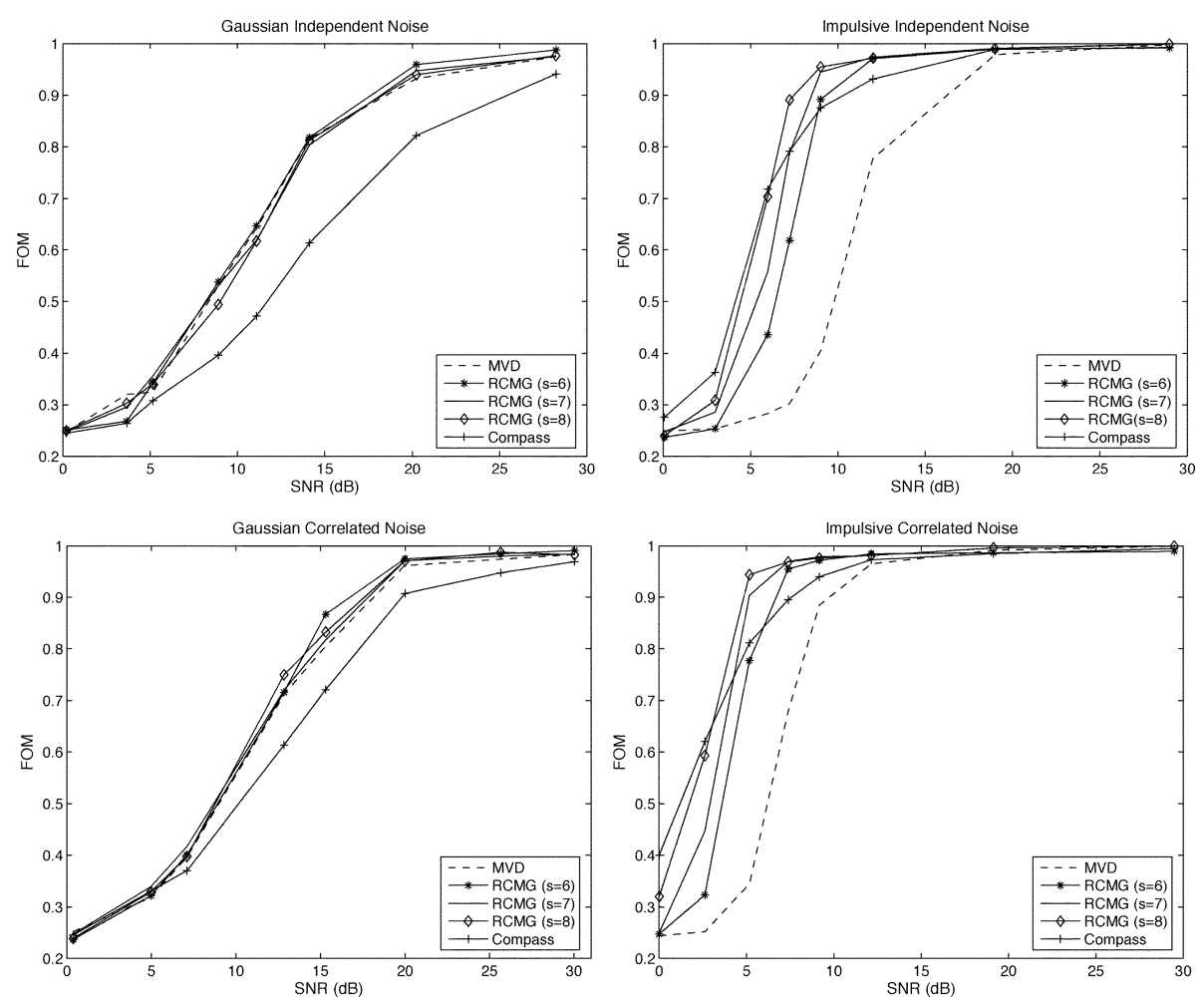

Fig. 11. FOM results for the Peppers image produced by RCMG, MVD, and Compass color edge detectors.

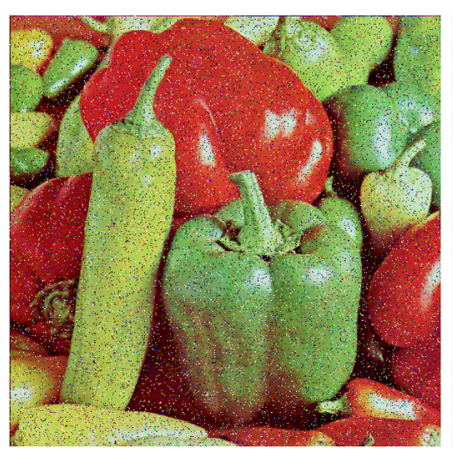

(a)

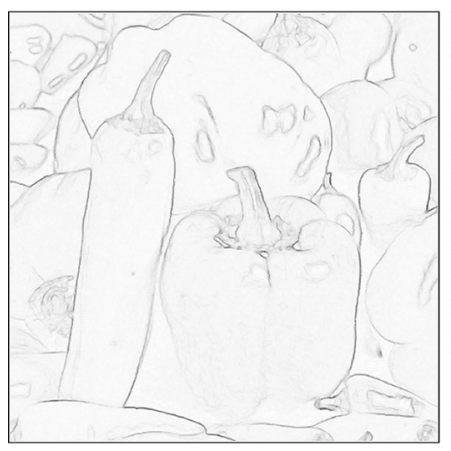

(c)

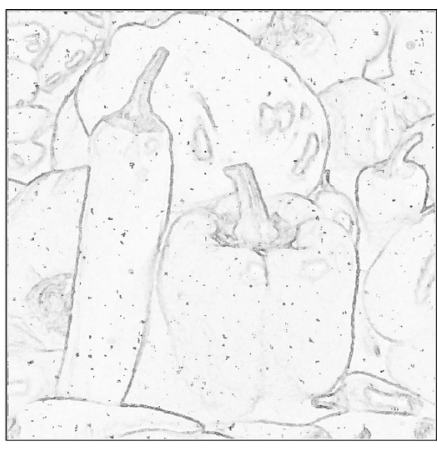

(b)

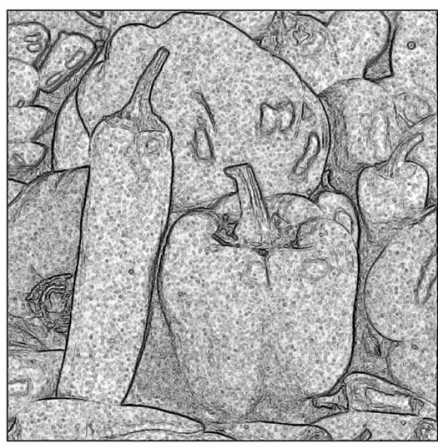

(d)

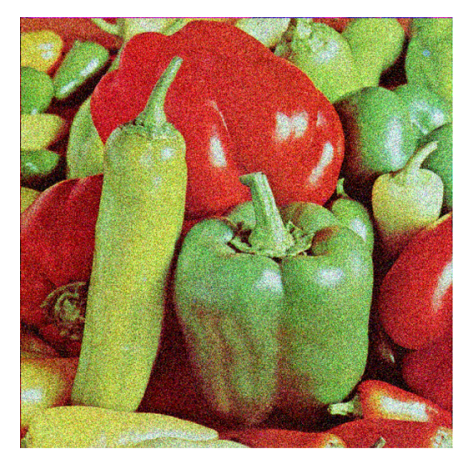

(a)

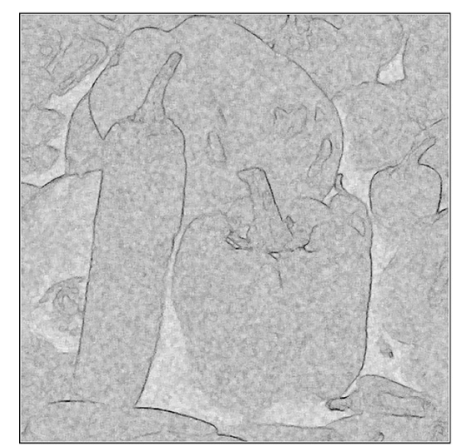

(c)

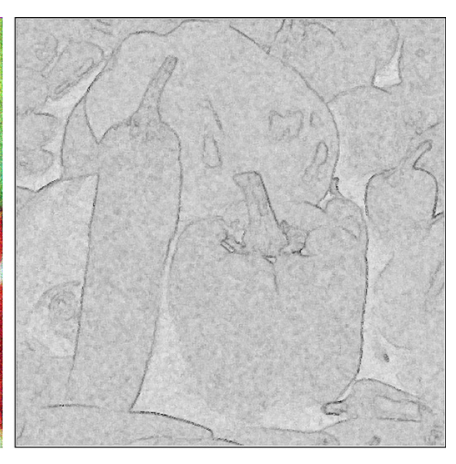

(b)

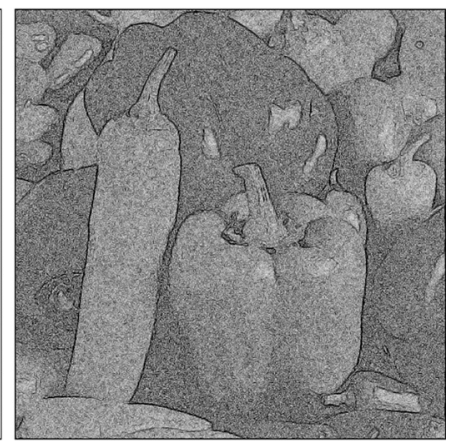

(d)

Fig. 12. Color edge results for the Peppers image corrupted by $10 \%$ correlated ( $\rho=0.5$ ) impulsive noise. (a) Original image. (b) MVD result. (c) RCMG result. (d) Compass result. (Color version available online at http://ieeexplore.ieee.org.)

they offer good performance with relatively low computation cost. In addition, the techniques can directly be applied to other

Fig. 13. Color edge results for the Peppers image corrupted by correlated Gaussian noise $(\sigma=10)$. (a) Original image. (b) MVD result. (c) RCMG result. (d) Compass result. (Color version available online at http://ieeexplore.ieee.org.)

vector-valued images such as those produced by multispectral imaging systems. 


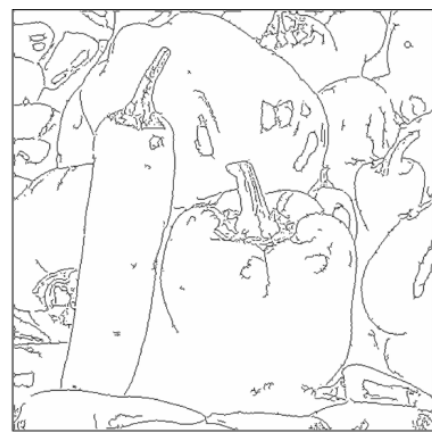

(a)

(c)

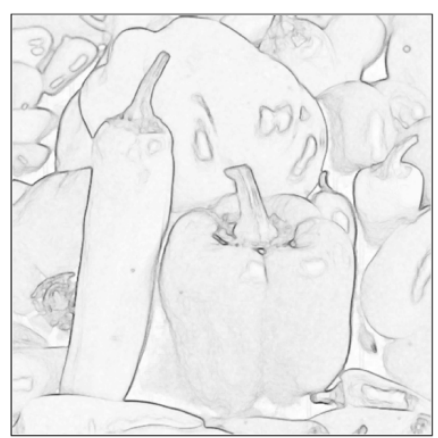

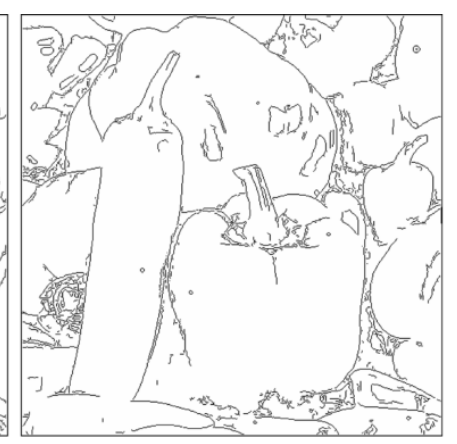

(b)

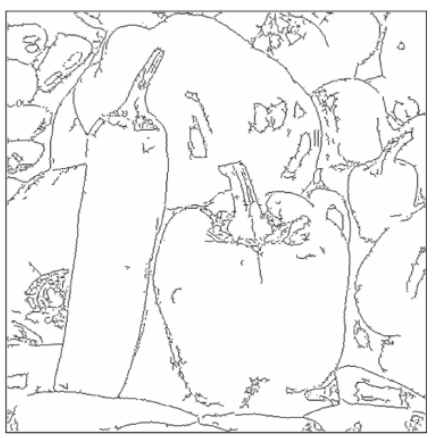

(d)
Fig. 14. Thresholded color edge results for the Peppers image using nonmaximal suppression and thresholding with hysteresis. (a) RCMG result. (b) Compass result. (c) RCMG gradient using combined distance metric. (d) Edge points from (c).

\section{REFERENCES}

[1] C. L. Novak and S. A. Shafer, "Color edge detection," in Proc. DARPA Image Understanding Workshop, vol. I, 1987, pp. 35-37.

[2] J.-F. Rivest, P. Soille, and S. Beucher, "Morphological gradients," J. Electron. Imag., vol. 2, no. 4, pp. 326-336, 1993.

[3] M. C. d'Ornellas, "A multiscale gradient approach for color-based morphological segmentation," in Proc. Int. Conf. Pattern Recognit., vol. 3, 2000, pp. 363-366.

[4] P. E. Trahanias and A. N. Venetsanopoulos, "Color edge detection using vector order statistics," IEEE Trans. Image Process., vol. 2, no. 2, pp. 259-264, Feb. 1993.

[5] - "Vector order statistics operators as color edge detectors," IEEE Trans. Syst., Man, Cybern. B, Cybern., vol. 26, no. 1, pp. 135-143, Feb. 1996.

[6] M. A. Ruzon and C. Tomasi, "Edge, junction, and corner detection using color distributions," IEEE Trans. Pattern Anal. Mach. Intell., vol. 23, no. 11, pp. 1281-1295, Nov. 2001.

[7] J. S. Lee, R. M. Haralick, and L. G. Shapiro, "Morphologic edge detection," IEEE Trans. Robot. Autom., vol. 3, no. 2, pp. 142-156, Apr. 1987.

[8] A. N. Evans, "Morphological gradient operators for color images," in IEEE Int. Conf. Image Process., vol. III, 2004, pp. 3089-3092.

[9] J. F. Canny, "A computational approach to edge detection," IEEE Trans. Pattern Anal. Mach. Intell., vol. 8, no. 6, pp. 679-698, Jun. 1986.

[10] I. E. Abdou and W. K. Pratt, "Quantitative design and evaluation of enhancement/thresholding edge detectors," Proc. IEEE, vol. 67, no. 5, pp. 753-763, May 1979.

[11] L. Lucchese and S. K. Mitra, "Color image segmentation: a state-ofthe-art survey," in Proc. Ind. Nat. Sci. Acad. (INSA-A), vol. 67 A, 2001, pp. 207-221.
[12] M. Heddley and H. Yan, "Segmentation of color images using spatial and color space information," J. Electron. Imag., vol. 1, no. 4, pp. 374-380, 1992.

[13] J. Fan, D. K. Y. Yau, A. K. Elmagarmid, and W. G. Aref, "Automatic image segmentation by integrating color-edge extraction and seeded region growing," IEEE Trans. Image Process., vol. 10, no. 10, pp. 1454-1466, Oct. 2001.

[14] T. Carron and P. Lambert, "Color edge detector using jointly hue, saturation and intensity," in Proc. IEEE Int. Conf. Image Process., vol. III, 1994, pp. 977-981.

[15] - "Fuzzy color edge extraction by inference rules quantitative study and evaluation of performances," in Proc. IEEE Int. Conf. Image Process., vol. II, 1995, pp. 181-184.

[16] S. Wesolkowski, M. E. Jernigan, and R. D. Dony, "Comparison of color image edge detectors in multiple color spaces," in Proc. IEEE Int. Conf. Image Process., vol. II, 2000, pp. 796-799.

[17] G. Robinson, "Color edge detection," Opt. Eng., vol. 16, no. 5, pp. 479-484, Sep. 1977.

[18] S. Di Zenzo, "A note on the gradient of a multiimage," Comput. Vis. Graph. Image Process., vol. 33, no. 1, pp. 116-125, Jan. 1986.

[19] A. Cumani, "Edge detection in multispectral images," Graph. Models Image Process., vol. 53, no. 1, pp. 40-51, 1991.

[20] M. Chapron, "A color edge detector based on statistical rupture tests," in Proc. IEEE Int. Conf. Image Process., vol. II, 2000, pp. 820-823.

[21] W. Alshatti and P. Lambert, "Using eigenvectors of a vector field for deriving a second directional derivative operator for color images," in Proc. 5th Int. Conf. Comput. Anal. Images Patterns, vol. 719, 1993, pp. 149-156.

[22] A. Koschan, "A comparative study on color edge detection," in Proc. 2nd Asian Conf. Comput. Vis., vol. III, 1995, pp. 574-578.

[23] M. Chapron, "A color edge detector based on Dempster-Shafer theory," in Proc. IEEE Int. Conf. Image Process., vol. II, 2000, pp. 812-815.

[24] - "A color edge detector based on abrupt change techniques," in Proc. IEEE Int. Conf. Image Process., vol. III, 1997, pp. 18-21.

[25] D. Zugaj and V. Lattuati, "A new approach of color images segmentation based on fusing region and edge segmentations outputs," Pattern Recognit., vol. 31, no. 2, pp. 105-113, Feb. 1998.

[26] A. Fotinos, G. Economou, and S. Fotopoulos, "Use of relative entropy in color edge detection," Electron. Lett., vol. 35, no. 18, pp. 1532-1534, 1999.

[27] F. Wen, M. Hu, and B. Yuan, "A comparative study on color edge detection," in Proc. IEEE Region 10 Conf. Comput., Commun., Contr. Power Eng., vol. I, 2002, pp. 511-514.

[28] S. Wesolkowski and M. E. Jernigan, "Color edge detection in RGB using jointly euclidean distance and vector angle," in Proc. IAPR Vis. Interf., 1999, pp. 1-9.

[29] L. Shafarenko, M. Petrou, and J. V. Kittler, "Automatic watershed segmentation of randomly textured color images," IEEE Trans. Image Process., vol. 6, no. 11, pp. 1530-1544, Nov. 1997.

[30] P. E. Trahanias and A. N. Venetsanopoulos, "Vector directional filters - a new class of multichannel image processing filters," IEEE Trans. Image Process., vol. 2, no. 4, pp. 528-534, Apr. 1993.

[31] D. Androutsos, K. N. Plataniotis, and A. N. Venetsanopoulos, "Distance measures for color image retrieval," in Proc. IEEE Int. Conf. Image Process., vol. II, 1998, pp. 770-774.

[32] P. Androutsos, D. Androutsos, K. N. Plataniotis, and A. N. Venetsanopoulos, "Color edge detectors: an overview and comparison," in Proc. IEEE Canad. Conf. Elect. Comput. Eng., vol. II, 1997, pp. 607-610.

Adrian N. Evans, photograph and biography not available at the time of publication.

Xin U. Liu, photograph and biography not available at the time of publication. 